\title{
Twentieth meeting.
}

Rockefeller Institute for Medical Research. February 20, 1907. President Flexner in the chair.

$$
26 \text { (I69) }
$$

Experimental studies on nuclear and cell division.

\section{By EDWIN G. CONKLIN.}

\section{[From the Zoological Laboratory, University of Pennsylvania.]}

During several seasons extensive experiments were made on the segmenting eggs of Crepidula plana. These experiments included a study of the influence on nuclear and cell division of hypertonic and hypotonic sea water, of ether, alcohol, etc., of the lack of oxygen, of the electric current, and of pressure and shaking. The following general conclusions may be drawn from this work :

I. Under the same treatment the effects may be extremely varied, owing, probably, to the different stages of cell division acted upon.

2. A dividing cell is much more easily disturbed or rendered abnormal than is a resting one; the mitotic figure in particular is very easily altered and most of the abnormalities observed arise from this source.

3. The earlier stages of cleavage are much more easily altered than are the later ones.

4. Certain general abnormalities occur after the most varied treatment, e.g., the general results both of concentration and of dilution of sea water are to produce polyasters and to prevent the cleavage of the yolk.

5. On the whole the results of the hypertonic solutions are the same whether they are produced by evaporation of the sea water or by the addition of $\mathrm{NaCl}, \mathrm{MgCl}_{2}$, or $\mathrm{KCl}$ to sea water ; in short, these salts exert no specific action on cell division. 
6. The most general modification of the mitotic figure is the production of polyasters, multipolar spindles, and as a consequence, multiple nuclei. In many cases the cells are filled with asters and irregular mitotic figures, during division, while in the resting stage they are filled with equally numerous resting centrosomes and nuclei.

7. The movements of the chromosomes are in many cases interrupted, so that they remain scattered along the spindle, while the cytoplasmic movements are frequently stopped or altered.

8. In some cases the achromatic portion of the nucleus is separated from the chromatic part, and the two may persist side by side during the resting stage of the cell ; in the division stages the achromatic nuclei give rise to asters, the chromatic to chromosomes and both may divide indefinitely, giving rise to large numbers of chromatic and achromatic nuclei.

9. The most general modification of the division of the cellbody is the suppression of the cleavage of the yolk; this occurs in practically all the experiments; at the same time the cleavage may proceed more or less regularly in the protoplasmic portion of the egg. In normal eggs the first and second cleavages divide the yolk into four equal cells (the macromeres) and, from each of these, three small cells (the micromeres) are budded off.

IO. If the yolk remains undivided it gives rise in certain cases to three micromeres, which have the characteristics of those formed from each of the four macromeres of the normal egg. If the yolk has divided once so as to form two macromeres, each of these may give rise to three micromeres, having the characteristics of the three quartet cells of the normal egg. In short, the number of micromeres depends upon the number of macromeres. When there are four of these as in normal eggs, the micromeres are formed in three quartets; when there are two, they are formed in three pairs; when there is but one macromere, i.e., when the yolk remains undivided, the micromeres are formed singly.

II. When eggs are subjected to pressure the third cleavage which normally gives rise to the first group of micromeres, may divide one or more of the macromeres equally, thus giving rise to five, six, seven or eight macromeres. If the pressure is removed from such eggs each macromere gives rise to three micromeres in 
a manner approximately normal ; again showing that the number of micromeres which may come from a macromere is constant, whatever the number of macromeres may be.

12. The results stated in the two preceding paragraphs show that the omission or the addition of cleavages does not alter the character or localization of the egg substances and that this localization, when unimpeded, determines the character of the cell division.

1 3. Isolated blastomeres undergo partial development, each giving rise only to the cells which it would form if still a part of the entire egg, but the general form of the cleavage mass is entire, $i . e$., there is no open side.

I4. A weak electric current destroys spindle fibers and astral rays, or prevents their formation and thus stops mitosis. It also destroys the polarity of the cell, prevents the separation of protoplasm and yolk, and may cause nuclei to migrate through the cell from one pole to another.

15. Abnormalities of mitosis may perpetuate themselves in subsequent divisions, even when the cause which first induced them is removed.

$27(170)$

\section{Heterotransplantation of blood vessels.}

By ALEXIS CarReL.

[From the Rockefeller Institute for Medical Research.]

It is well known that the tissues of an animal do not grow or grow hardly at all in an animal of another species. Nevertheless, I attempted to transplant to cats, blood vessels resected from dogs, with the aim of ascertaining whether the vessels in spite of the toxic action of the cat's blood on the dog's tissue, could take over the functions of the vessels removed.

The method consisted of removing a segment of the abdominal aorta of a cat, and of reestablishing the circulation in the lower part of the aorta by interposing a segment of the jugular or carotid of a dog and suturing it to the cut ends of the aorta. 